\section{Meningeom und Rauchen: Frauen im Vorteil}

Frauen, die rauchen oder früher geraucht haben, tragen ein um $20 \%$ niedrigeres Risiko, an einem intrakraniellen Meningeom zu erkranken. Für Männer hingegen erhöht der Tabakkonsum die Meningeomgefahr.

D en Zusammenhang zwischen Rauchen und Meningeom-Risiko haben US-Epidemiologen in einer populationsbasierten Fall-Kontroll-Studie untersucht. Teilnehmer waren 1.433 Patienten im Alter von 29 bis 79 Jahren, bei denen zwischen Mai 2006 und April 2011 ein Meningeom diagnostiziert worden war. Die Vergleichsgruppe bestand aus 1.349 Personen, die mit den Patienten nach Alter und Geschlecht gematcht waren.

Für Raucherinnen bzw. Ex-Raucherinnen ergab sich dabei ein um $20 \%$ reduziertes Risiko für eine MeningeomDiagnose. Männliche (Ex-)Raucher wiesen hingegen ein um $30 \%$ höheres Risiko auf. Bestätigt wurde dieses Resultat durch eine zusätzliche Metaanalyse von sechs Studien zum Thema Meningeom, in die Daten von 2.614 Patienten und 1.179.686 Kontrollpersonen einflossen. Die dem Rauchen geschuldete Risikoreduktion lag hier für Frauen bei $18 \%$, die Risikosteigerung für Männer betrug 39\%.

Fazit: (Ex-)Raucherinnen erkranken seltener an Meningeomen. Männer hingegen steigern durch Tabakgenuss die Wahrscheinlichkeit, einen solchen Hirntumor zu entwickeln. Als Ursache werden nicht zuletzt hormonelle Faktoren diskutiert: So hat Zigarettenrauch antiöstrogene Wir-

\title{
Übergewicht bei Raucherinnen mit geringerem Lungenkrebsrisiko verbunden
}

\begin{abstract}
Wer im Verhältnis zu seiner Größe viele Pfunde auf die Waage bringt, muss vielleicht kardiovaskuläre Erkrankungen, Gelenkschmerzen und unter Umständen auch Darm- oder Schilddrüsenkrebs fürchten, aber: Mit zunehmendem Body-Mass-Index (BMI) sank in einer Studie das Lungenkrebsrisiko.
\end{abstract}

\footnotetext{
lewellyn Smith und sein Team vom

- National Cancer Institute in Rockville, MD/USA, hatten knapp 450.000 Männer und Frauen im Alter zwischen 50 und 71 Jahren über fast zehn Jahre beobachtet; in diesem Zeitraum hatten 9.400 Lungenkrebs entwickelt, darunter gut 400 Nichtraucher. Der mittlere BMI betrug bei den Männern 26,6, bei den Frauen $25,8 \mathrm{~kg} / \mathrm{m}^{2}$. Übergewichtig war fast die Hälfte der Männer sowie ein knappes Drittel der Frauen, als adipös wurde jeweils ein knappes Fünftel eingestuft.

Der inverse Zusammenhang des BMI mit dem Krebsrisiko war bei Teilnehmern beiderlei Geschlechts signifikant, bei den Frauen sogar noch etwas stärker ausgeprägt. Besonders erstaunlich: Der Effekt beschränkte sich auf Raucher, auch solche, die früher geraucht hatten und aktuell entwöhnt waren. In der (Ex-)RaucherGruppe war es zudem unerheblich, ob und wann sie mit dem Rauchen aufgehört
}

hatten und wie viele Zigaretten sie am Tag geraucht hatten. Für die Nichtraucher galt das Prinzip ,je dicker, desto geringer die Lungenkrebsgefahr" dagegen nicht.

Um zu vermeiden, dass das Ergebnis durch unentdeckte Lungenkarzinome verfälscht wurde, die zu einer Gewichtsreduktion hätten führen können, hatten die Forscher in einer Subgruppenanalyse alle Teilnehmer ausgeschlossen, bei denen in den ersten sieben Jahren nach Studienbeginn ein Lungenkrebs diagnostiziert worden war. Von den verbleibenden Teilnehmern hatten Männer mit einem BMI über 30 ein um $9 \%$ geringeres Krebsrisiko als schlankere Geschlechtsgenossen mit Werten zwischen 22,5 und 25. Bei den Frauen verhielt es sich ähnlich, nur war das Risiko bei den korpulenteren Teilnehmerinnen sogar um $16 \%$ reduziert. Betrachtete man das histologische Staging, zeigte sich, dass der Zusammenhang bei Männern nur für Adenokarzinome galt, kungen, indem er den Metabolismus von Östradiol zu inaktiven Katechoöstrogenen fördert. Bekannt ist auch, dass andere hormonabhängige Tumoren, wie das Endometriumkarzinom, bei Raucherinnen seltener zu finden sind. Ob die niedrigere Meningeom-Häufigkeit bei Raucherinnen tatsächlich hormonelle Ursachen hat, muss allerdings noch eingehender geprüft werden.

Robert Bublak

Claus EB et al. Cigarette smoking and risk of meningioma: the effect of gender. Cancer Epidemiol Biomarkers Prev. 2012 Apr 11. [Epub ahead of print].

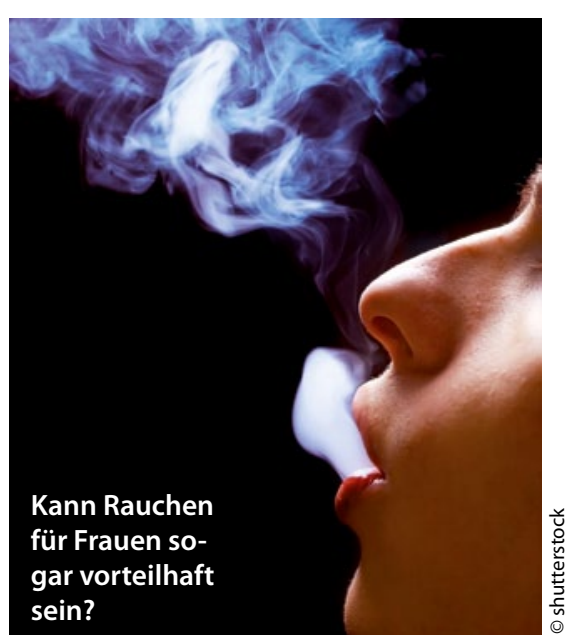

bei Frauen dagegen für Adeno- und Plattenepithelkarzinome.

Die Forscher spekulieren, dass die bei Adipositas vermehrt gebildeten Östrogene möglicherweise an Schutzmechanismen beteiligt sein könnten. Hierzu passt, dass im Hinblick auf das Lungenkrebsrisiko vor allem Frauen von einem hohen BMI profitierten.

Fazit: Zwischen dem Body-Mass-Index und dem Risiko, Lungenkrebs zu entwickeln, besteht nach diesen Ergebnissen ein inverser Zusammenhang. Überraschenderweise waren es vor allem (Ex-) Raucherinnen, bei denen das Übergewicht mit einem geringeren Krebsrisiko verbunden war. Die Ursache für dieses Phänomen bleibt unklar. Elke Oberhofer

Smith $L$ et al. Body mass index and risk of lung cancer among never, former, and current smokers. J Natl Cancer Inst. 2012 Mar 27. [Epub ahead of print]. 